\title{
STIMULATED LONGITUDINAL EMITTANCE GROWTH IN THE MAIN RING
}

\author{
G. Jackson and T. Ieiri 1 \\ Fermi National Accelerator Laboratory 2 , Box 500, Batavia, Il 60510
}

\section{Abstract}

During fixed target operations beam intensity is limited by coherent instabilities in both the Main Ring and Tevatron. The growth rates for instabilities are generally inversely proportional to the proton bunch length. Since fixed target operations are insensitive to the longitudinal emittance of the beams, bunch spreaders are employed to increase the emittance, and hence the bunch length. Emittance growth is stimulated by injecting noise onto either the RF phase or amplitude control voltages. Test results of the efficiency of various stimulation schemes are reported. The design of a bunch length monitor, used to measure the effect of the bunch spreader, is also presented.

\section{Motivation}

During fixed target physics operations the Fermilab accelerators are expected to transmit as many protons as possible to $800 \mathrm{GeV}$. The maximum proton intensity, which in the last $1987 / 1988$ fixed target run was around $1.8 \times 10^{13}$ protons/pulse, or about $1.8 \times 10^{10}$ protons/bunch. This limit was imposed by the simultaneous onset of instabilities in the Main Ring and the Tevatron. The Main Ring problem is quite erratic, and has not been identified. It strikes at the peak Main Ring energy of $150 \mathrm{GeV}$, just before the beam is transferred into the Tevatron. The Tevatron suffers from a slow head-tail instability [3] which begins soon after the beam energy has exceeded $600 \mathrm{GeV}$.

The threshold intensity of this instability was a function of longitudinal emittance [4]. The peak intensity of $1.8 \times 10^{10}$ protons/bunch coincided with a $90 \%$ invariant longitudinal bunch area (A) of $5 \mathrm{eV}-\mathrm{sec}$. At an area of $1.5 \mathrm{eV}-\mathrm{sec}$ the peak intensity was $1.4 \times 10^{10}$ protons/bunch.

The larger bunch area of $5 \mathrm{eV}$-sec was stimulated by devices called bunch spreaders. Instead of spending time eliminating the instability, it was decided to increase the threshold instability intensity by increasing the bunch length by a proportional amount. The Main Ring bunch spreader was used both to increase the Main Ring instability threshold intensity, and to help the Tevatron bunch spreaders attain a final longitudinal emittance of $5 \mathrm{eV}$-sec.

This document describes efforts to upgrade the bunch spreaders in anticipation of even greater intensity requirements $\left(2-3 \times 10^{10}\right.$ protons/bunch) in the coming fixed target run. In order to better analyze their performance, a $10 \mathrm{kHz}$ bandwidth bunch length monitor has been built, and is also described below. The discussion will center around the new, recently tested Main Ring bunch spreader and bunch length monitor.

\section{Bunch Length Monitor}

A bunch length monitor is needed in order to measure the performance of a bunch spreader. Variation of the bunch length must be easily and clearly read from the monitor. The previous method of measuring the bunch length is to photograph the beam profile on a wide band resistive wall monitor. This method is too imprecise and to cumbersome. Instead, a real time monitor which outputs a voltage proportional to bunch length has been proposed [5] and developed.

Assuming a train bunches with Gaussian longitudinal density distributions of rms width $\sigma$, the Fourier transform of the response of a resistive wall monitor is

$$
V_{\mathrm{m}}=2 \mathrm{~V}_{\circ} \exp \left(-\frac{1}{2} \mathrm{~m}^{2} \omega_{\mathrm{rf}}^{2} \sigma^{2}\right),
$$

where $m>0$ and $V_{m}$ is the voltage per unit bandwidth at the mth harmonic of the $R F$ angular frequency $\omega_{\mathrm{rf}}$. Available is a stripline detector, and not a resistive wall monitor. Its length is such that the $m=2$ line is missing, so therefore the $m-1$ and $m-3$ voltages are detected. Basically, the monitor performs the analog calculation

$$
\sigma=\frac{1}{k} \sqrt{-\ln \left(v_{3} / v_{1}\right)},
$$

where $k$ is an empirically determined number whose theoretical value should be $2 \omega_{r f}$. In fact, the $95 \%$ interval of the beam distribution, referred to as the

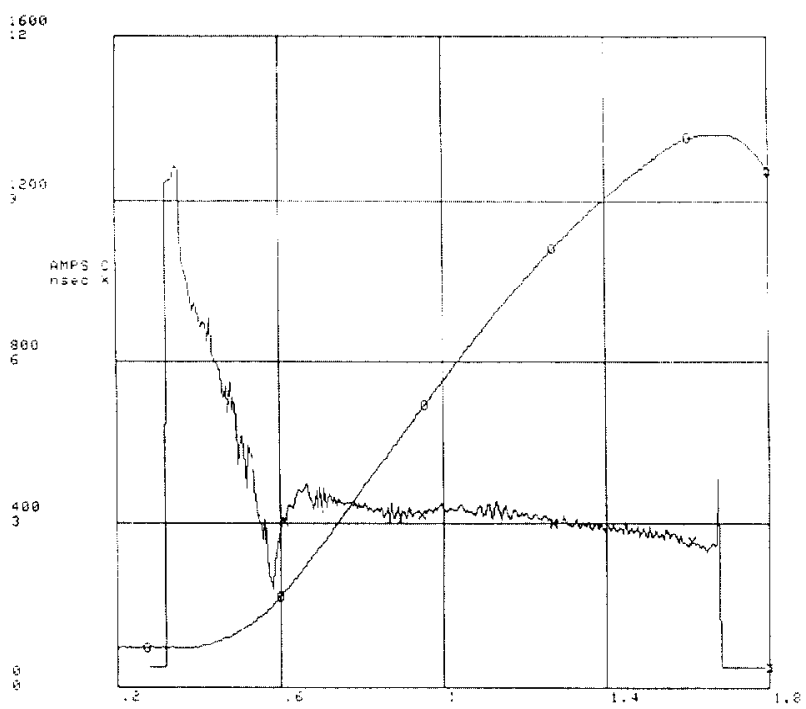

Figure 1: Measured Main Ring bunch length as a function of time. The curve marked with circles is the current in the dipole bus. The scales are 3 nsec and 0.4 seconds per division.

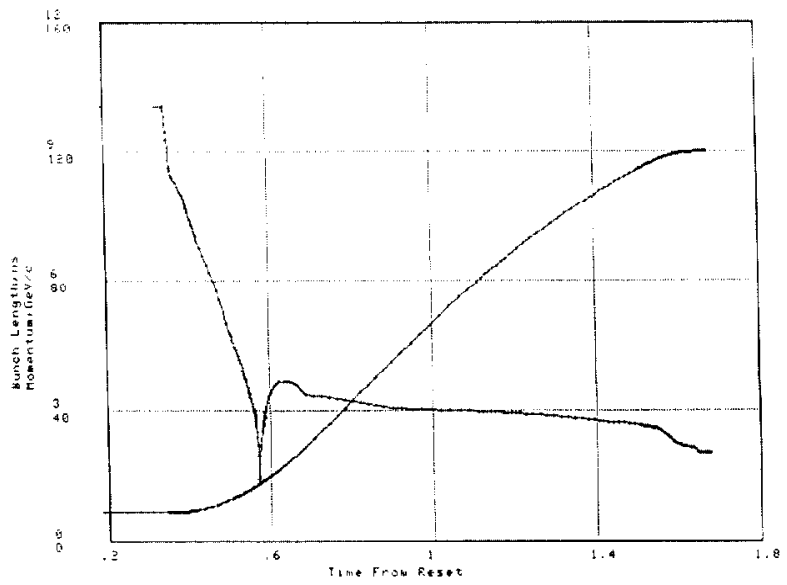

Figure 2: Expected Main Ring bunch length as a function of time. The beam momentum is also displayed. The scales are identical to figure 1. 
full bunch length, is the quantity of interest and the result given by the monitor. Calibration is accomplished through detailed comparison with bunch profile photographs.

The output of the stripline detector is split and sent to two parallel circuits, one to detect the $53 \mathrm{MHz}$ component of the beam and the other to detect the $159 \mathrm{MHz}$ component of the beam. Using matched $1 \mathrm{MHz}$ bandwidth bandpass filters centered about these two frequencies, the monitor output is insensitive to the number of bunches in the accelerator. Heterodyne receivers then convert the $\mathrm{RF}$ amplitudes into $10 \mathrm{kHz}$ bandwidth signals wich are manipulated according to equation (2). The dynamic range of the system is $30 \mathrm{db}$, which means it can handle intensity fluctuations over a factor of 30 range.

Figure 1 shows the measured response of the Main Ring bunch length monitor, along with the dipole current, as a function of time in an antiproton production acceleration cycle. Injection of 81 bunches, with an intensity of approximately $1.8 \times 1010$ protons/bunch, takes place at 0.3 seconds. Transition [7], the point at wich the bunch length attains its minimum value, occurs just before 0.6 seconds into the cycle. Flattop is reached at 1.6 seconds, where the beam undergoes bunch rotation [6] just prior to extraction into the antiproton target. Figure 2 contains the expected full bunch length assuming a constant bunch area of $0.22 \mathrm{eV}$-sec. The agreement with the measured curve in figure 1 is very good. The noise in the measured bunch length is dominated by real beam bunch length fluctuations.

\section{Bunch Spreader Physics}

The invariant longitudinal emittance of a bunch can be changed through the introduction of random energy kicks to the beam [8]. Since narrow bunch lengths are to be avoided, and adiabatic bunch narrowing is taking place up the ramp, bunch spreading must occur while the beams are accelerating.

The bunch spreader can be connected to an RF phase shifter (as was the case in an old bunch spreader) or to the $\mathrm{RF}$ peak voltage control. The problem with phase modulation is the need to decrease the radial offset feedback gain, which determines the $R F$ frequency during acceleration. In the case of $R F$ voltage modulation and full radial position gain the beam energy increment per turn is conserved and the synchronous phase is modulated. As the voltage is decreased, the synchronous phase is increased toward 900. Therefore, the small amplitude voltage gradient is reduced by both voltage decrease and the $\cos \phi_{\mathrm{S}}$ dependence of the gradient on synchronous phase. Voltage modulation seems to be the theoretically best channel for introducing a bunch lengthening signal into the Main Ring RF system.

The dependence of the amplitude growth rate on the frequency content of the bunch spreader signal is very important. First, there is no growth in the amplitude of a proton by this mechanism unless some fraction of the spreader power spectrum is at twice the natural particle frequency. Second, there is a spread in the natural proton synchrotron frequencies for a finite length bunch. The functional dependence of the synchrotron period on oscillation amplitude is quite complicated, requiring evaluation of elliptic integrals [8]. For phase oscillation amplitudes $\phi$ about the synchronous phase angle $\phi_{\mathbf{S}}$ which are at most half of the distance between the unstable fixed point and synchronous phases, this functional dependence can be approximated by the relationship

$$
\Omega(\dot{\phi})=\Omega(0)\left(1-a^{2}\left(\phi_{s}\right) \phi^{2}\right)
$$

where $\phi$ and $\phi_{S}$ are in degrees,

$$
\alpha^{2}\left(\phi_{s}\right)=\beta_{0}+\beta_{2} \phi_{s}^{2}+\beta_{4} \phi_{s}^{4} \quad,
$$

and where $\beta_{0}=0.194 \times 10^{-4}, \quad \beta_{2}=0.194 \times 10^{-8}$, and $\beta_{4}=0.603 \times 10^{-11}[9]$. This approximation is best at zero synchronous angle [10], getting progressively worse as $\phi_{\mathrm{S}}=900$ is approached.

The limit to the amplitude of bunch spreading noise in the old bunch spreaders was determined by the onset of beam loss from the RF bucket, and hence from the accelerator. This loss was minimized by applying random noise on the $\mathrm{RF}$ voltage control at frequencies at twice the small amplitude synchrotron frequency. By constructing a sharp 8 -pole active filter network, the noise amplitude at half this frequency was reduced by $30 \mathrm{db}$. The idea is that only protons at small amplitudes are spread, and protons near the separatrix in longitudinal phase space are unmolested.

\section{New Bunch Spreader Design}

Since at transition energy the momentum aperture of the Main Ring is at its minimum, bunch spreading can only occur after transition. The synchrotron frequency, as well as the $R F$ voltage, are plotted in figure 3 . Note that after transition the synchrotron frequency varies substantially, and twice the synchrotron frequency ranges from 300 to $500 \mathrm{~Hz}$. Since beam losses must be minimized, a narrow band variable frequency noise source was required.

The noise is generated by amplifying and filtering the Johnson noise across a resistor. By mixing the noise and a voltage controlled reference frequency, a variable frequency noise band is created. After additional filtering, this noise signal is gated and amplitude modulated using AD532 analog multipliers. The resultant signal is summed with the $\mathrm{RF}$ amplitude control voltage and sent on to the $R F$ amplifiers.

A control voltage of 10 volts corresponds to approximately $4 \mathrm{WV}$ of peak RF voltage sampled by the beam. The noise bandwidth is $100 \mathrm{~Hz}$. Both the amplitude and frequency of the noise voltage are functions of time in the acceleration cycle through the use of ramp generators connected to the RF control system. Gating is accomplished through timing pulses also generated by the RF control system.

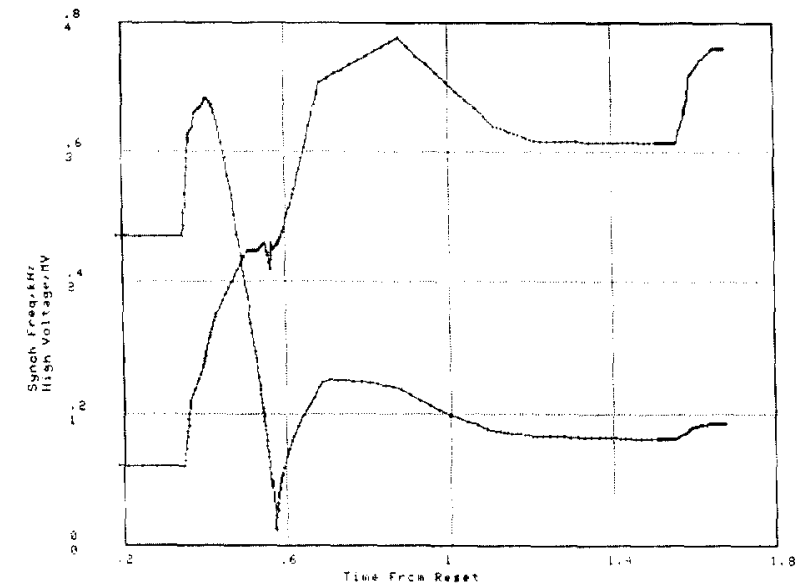

Figure 3: Predicted RF voltage and synchrotron frequency during a Main Ring acceleration cycle. The scales are $1 \mathrm{MV}$ and $200 \mathrm{~Hz}$ per division. As expected, the synchrotron frequency dips down to zero at transition.

\section{Measured Performance}

The new Main Ring bunch spreader has been tested under the same conditions shown in figure 1 . Figure 4 shows the measured bunch length, RF voltage, and total 
beam intensity in 81 bunches during the acceleration cycle with the bunch spreader activated. The bunch length curve should be compared with figure 1 , where the spreader was turned of $f$.

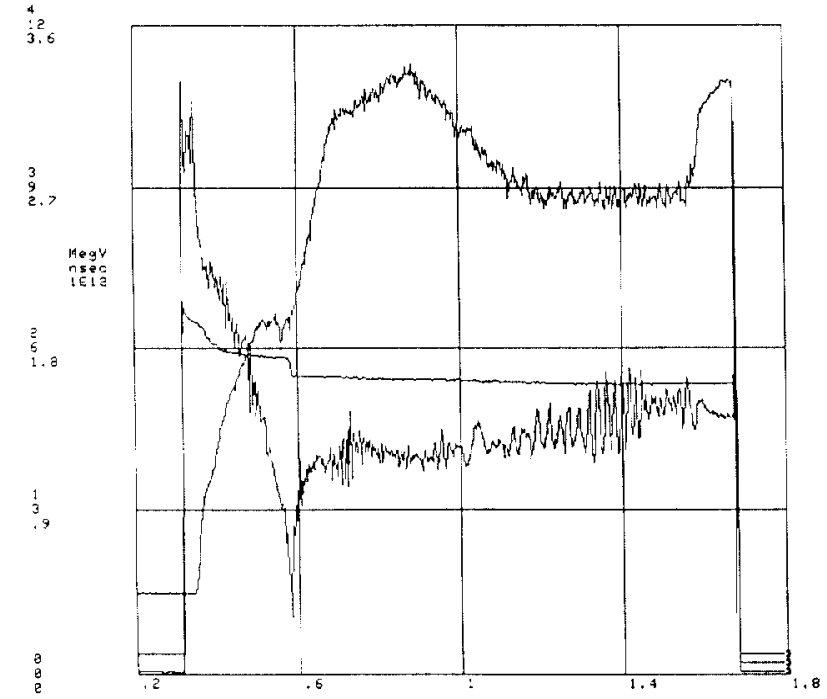

Figure 4: Measured performance of the new Main Ring bunch spreader. Plotted are the RF voltage, the bunch length, and the total beam intensity in 81 bunches. The scales are $1 \mathrm{MV}, 3 \mathrm{nsec}$, and $9 \times 10^{11}$ protons/beam per division, respectively.

By looking at the modulation on the measured RF voltage, it is clear that bunch spreading started just after transition and continued through the majority of the acceleration interval. The growth in the bunch length during this period is uneven, but with a sum total growth in bunch length of approximately $1.5 \mathrm{nsec}$, or $50 \%$.

On the other hand, even though the losses are dominated by the poor Main Ring lifetime at low momentum and the step loss through transition, some beam losses does occur due to the bunch spreader. With a total loss due to bunch spreading of around $1 \%$, a great improvement has been made over the performance of the old bunch spreader [9].

It should be pointed out that the frequency and amplitude curves used in this test have not been extensively optimized. During normal operations a considerable amount of real time fine tuning will be possible.

\section{Tevatron Design}

In the near future a Tevatron version of the bunch spreader will be constructed and installed. There will be two major differences between the Main Ring and Tevatron systems. First, as shown in figure 5, the synchrotron frequency during the Tevatron acceleration cycle is much lower than that in the Main Ring. Second, since the Tevatron RF system does not employ beam driven frequency or phase feedback systems, phase noise will be introduced at the synchrotron frequency, rather than $R F$ voltage noise at twice the synchrotron frequency.

The reason for trying phase noise at the synchrotron frequency is related to theoretical questions concerning the single proton response to the two types of RF noise. In the case of phase noise the response of the longitudinal emittance can be readily calculated via a harmonic oscillator solution, as was done in the Tevatron in the case of transverse emittance growth [11]. The growth rate of large amplitude particles, and hence their loss rate from the bucket, can be calculated. These calculations

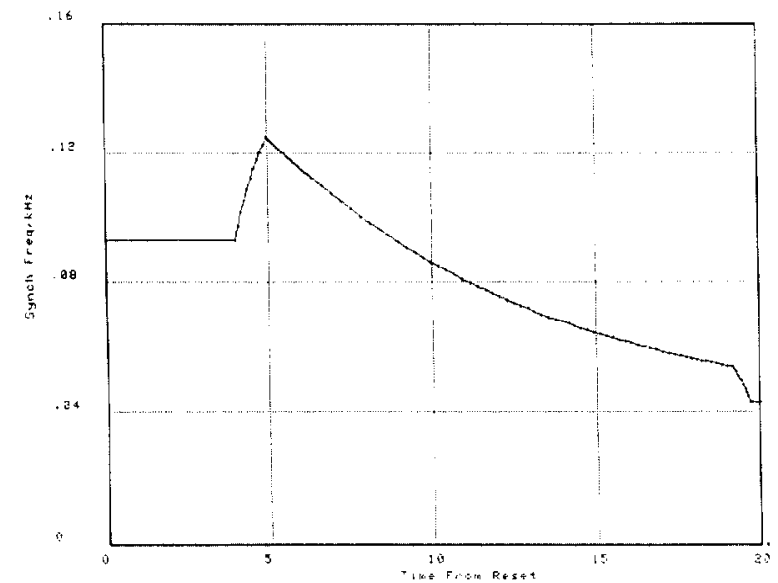

Figure 5: Calculated value of the small amplitude synchrotron frequency during Tevatron acceleration. The scales are 5 seconds and $40 \mathrm{~Hz}$ per division.

provide guidance as to the sharpness of the edges of the noise band in frequency space.

For RF voltage noise the dominant response of a proton is described by the differential equation

$$
\ddot{x}+w_{0}^{2}\left[1+\sum a \cos \left(2 \omega_{\alpha} t\right)\right] x=0 \text {. }
$$

In the case of a single modulation frequency the solutions of this differential equation are called Mathieu functions [12]. These functions are quite nonlinear, and more work is needed to understand the relationships between noise frequency and amplitude and beam loss for large amplitude protons.

\section{Conclusions}

In order to avoid intensity limits during fixed target operations, bunch spreaders arc used to stimulate longitudinal emittance growth. In order to avoid beam loss, narrow band random noise is applied near a multiple of the small amplitude synchrotron frequency during acceleration.

A new Main Ring bunch spreader has been tested which allows time dependent noise frequency and amplitude. Tests show that $50 \%$ bunch length growths are possible with tolerable beam loss.

Theoretical work aimed at trying to understand the difference between voltage and phase noise is underway. While the Main Ring bunch spreaders use RF voltage noise at twice the synchrotron frequency, the new Tevatron system will attempt to use phase noise at the synchrotron frequency itself.

\section{References}

1. Address is KEK, Tsukuba, Ibaraki, 305, Japan

2. Operated by the Universities Research Association under contract with the U.S. Department of Energy.

3. S.A. Bogacz, M. Harrison, and K.Y. Ng, Fermilab note FN-485 (1988).

4. S.A. Bogacz, Fermilab note FN-498 (1988).

5. R. Siemann, Private communication.

6. J. Griffin, et. al., IEEE Trans. Nucl. Sci. NS-30, 2630 (1983).

7. E. Courant and H. Snyder, Ann. Phys. 3,1 (1958).

8. G. Dome, CERN 84-15, 215 (1984).

9. G. Jackson, Fermilab note TM-1572 (1989).

10. S. Ohnuma, Fermilab note TM-1381 (1986).

11. S.R. Mane and G. Jackson, Nucl. Inst. and Meth. A, in press (1989).

12. M. Abramowitz and I. Stegun, Handbook of Mathematical Functions, Dover Publications, Inc., New York (1972), pg.721. 